Running head: RISK DATA

Cues and knowledge structures used by mental-health professionals when making risk assessments

Christopher D. Buckingham

Computer Science, Aston University

Ann Adams

Warwick Medical School, University of Warwick

Chris Mace

Department of Psychology, University of Warwick

5082 words (excluding abstract)

Corresponding author: C.D.Buckingham, Computer Science, Aston University, Aston

Triangle, Birmingham, B47ET.

email: c.d.buckingham@aston.ac.uk

Tel: 0121-204-3450; Fax: 0121-204-3681

The work was carried out at Aston and Warwick Universities 


\title{
Cues and knowledge structures used by mental-health professionals when making risk assessments
}

\begin{abstract}
Background: Research into mental-health risks has tended to focus on epidemiological approaches and to consider pieces of evidence in isolation. Less is known about the particular factors and their patterns of occurrence that influence clinicians' risk judgements in practice. Aims: To identify the cues used by clinicians to make risk judgements and to explore how these combine within clinicians' psychological representations of suicide, self-harm, self-neglect, and harm to others.

Method: Content analysis was applied to semi-structured interviews conducted with 46 practitioners from various mental-health disciplines, using mind maps to represent the hierarchical relationships of data and concepts.

Results: Strong consensus between experts meant their knowledge could be integrated into a single hierarchical structure for each risk. This revealed contrasting emphases between data and concepts underpinning risks, including: reflection and forethought for suicide; motivation for self-harm; situation and context for harm to others; and current presentation for self-neglect. Conclusions: Analysis of experts' risk-assessment knowledge identified influential cues and their relationships to risks. It can inform development of valid risk-screening decision support systems that combine actuarial evidence with clinical expertise.

Declaration of interest: This research was funded by a NHS NEAT grant.
\end{abstract}

\section{Introduction}

Risk assessment is an essential skill for mental-health professionals but its scientific basis remains imprecise. This is partly due to the relative rarity of risk events like suicide or violence among psychiatric populations (Owens, Wood, Greenwood, Hughes, \& Dennis, 2005; Szmukler, 2001) and partly because of the difficulties with studying many of the factors that 
influence clinical judgements in practice. Actuarial approaches to risk prediction are preferred because of claims they are evidence-based (Bouch \& Marshall, 2005), although their usefulness to specific cases has been questioned (Godin, 2004; Holdsworth \& Dodgson, 2003). Furthermore, they tend to emphasise relatively enduring "static" factors over transient "dynamic" ones, even though the latter are much more important in clinical practice (Maden, 2003).

The key to clinical risk judgements is an individual patient's qualitative and idiosyncratic cues (Holdsworth \& Dodgson, 2003) and overemphasis of actuarial data can be seen as "dehumanising" (Godin, 2004). The pattern of cues are often significant rather than their isolated effects. For example, the risk associated with young, single mothers is greater than the risk accumulated by considering the cues of gender, parental status, marital status, and age separately. The snag is that there are myriads of potential patterns residing in the cues and empirical demonstrations of their effects are extremely difficult to conduct, which is why most evidence pertains to individual factors (e.g. Skegg, 2005; Hanson, 2005; Castle, Duberstein, Meldrum, Conner, \& Conwell, 2004).

Nevertheless, risk assessments would be improved by the systematic incorporation of the full range of cues considered by clinicians. Unfortunately, there is little agreement on what this should be (Higgins, Watts, Bindman, Slade, \& Thornicroft, 2005), or how cues might be considered in combination (Monahan et al., 2000). It is a major reason why there is no single approved approach and it motivated Watts et al. (2004) to develop the Clinical Assessment of Risk Decision Support (CARDS), which attempted to integrate both evidence-based and clinical factors. Qualitative methods were used, such as workshops, focus groups, and Delphi consultations, to select risk data from factors identified in the literature or that emerged through discussions with participants. The research reported in this paper also adopted a qualitative approach to identify risk data but differs by focussing on how clinicians conceptualise risk knowledge. The aim is to elicit the psychological knowledge structures used by clinicians and expose interesting patterns of information.

The paper begins by describing the methods used, with an emphasis on how they helped 
integrate risk-assessment knowledge elicited from the sample of clinicians. The resulting comprehensive and detailed model is then analysed to show the levels of agreement existing between clinicians for the different elements of knowledge and to show how these elements vary across the risks. Implications for risk assessment are discussed and the paper concludes by considering how the knowledge structure can make a major contribution to the development of valid risk-screening decision support systems.

\section{Method}

\section{Sample}

Multi-Centre Research Ethics Committee clearance was obtained to permit participation from UK Trusts and 46 mental-health professionals were interviewed. Only those with at least two years post-qualification experience and in clinical practice at the time of the study were eligible for inclusion. Recruitment was purposive, targetting people using criteria such as their publication records, clinical profiles, and memberships of relevant committees, as well as asking clinical managers to select appropriate people. The result was a panel of experts covering multiple perspectives and experiences relating to risk assessment, ranging across clinical disciplines, adult mental health services, and professional contexts (e.g. NHS, academic), including forensic specialties. Most of the participants were from psychiatric nursing (21) and psychiatry (14), but social workers (3), general practitioners (3), and psychologists (5) were also represented.

\section{Interviews}

Previous pilot work (Buckingham \& Chan, 2002) identified four main risks that are the focus of assessments by mental-health professionals: suicide, self-harm $(\mathrm{S}-\mathrm{H})$, harm to others (HTO), and self-neglect (S-N). Although the risks were not explicitly defined for the experts in the current study, the understanding used for subsequent coding of interviews was that suicide included self-harming actions with the intent of causing death, compared to S-H where there was no intention of dying; HTO covered violence, aggression, abuse, and gross neglect of other 
people; and S-N meant the inability to care for oneself or take the necessary actions to avoid $\mathrm{S}-\mathrm{H}$.

These risks and their main underlying factors, which the pilot work also identified, delineated the ground interviews needed to cover in order to elicit relevant knowledge structures. They were used as prompts to stimulate further information if required, within interviews that began with an open invitation to define the areas of greatest significance to each participant. The initial question asked experts to imagine they were in their normal clinical environment and assessing the risks associated with people who are presenting with mental-health difficulties: what are the most important factors to be considered? The idea was for them to conjure up generic presentations of different risks as well as specific cases that might naturally come to mind during the interview. The interviewer tried to pursue expert's own thought trains rather than direct them, with neutral prompts used to ensure full explication of factors previously mentioned.

\section{Content analysis of interviews}

Insert Figure 1 about here

Interviews were audiotaped, transcribed, and subjected to a form of content or thematic analysis (Neuendorf, 2002) that aimed to identify the concepts associated with risk assessments and their constituent components. Our pilot work enabled us to produce a coding framework for capturing information about each risk, which was amended and improved by the initial analyses of interviews in the current research. This coding framework was represented as a mind map (Buzan, 2003) and used as the template for coding each expert's interview (Figure 1).

Mind maps are visual representations of the inherent structure within people's knowledge, where the main idea is placed in the central "node" of the map. This focal node is "risk" in Figure 1, where the blank space in the name allows the specific risk to be entered. Mental-health risks have a number of subconcepts relating to them, which are the immediate 
branches (nodes) off the central node in Figure 1, such as history, social context, assessment, etc. Each of these may be further subdivided into subconcepts, such as client episodes and family for the history concept. As the mind map spreads out from the central idea, the concepts become more and more specific and detailed, effectively defining a hierarchical knowledge structure. If one imagines holding up the central node, the rest would hang down like an upended tree, with the branches ending in leaves that represent the lowest level data pertaining to risk; they are the most concrete or tangible cues used in risk assessments.

Mind-maps were recorded for each interview using Freemind (Freemind, 2006), an open-source software program. Transcript line numbers where particular factors were mentioned (e.g. "triggers") were recorded after corresponding node names on the template (e.g. at the top right of Figure 1 for triggers), or by adding further nodes to it if the factor did not already appear. Any template nodes without associated line numbers were then deleted to produce a mind map that captures the inherent structuring of expert's knowledge, as communicated in the interview. Agreement between the decisions of three independent coders was better than $90 \%$ using the mind-map framework for coding individual interviews in this way.

Insert Figure 2 about here

The next stage of analysis integrated each individual mind map into a combined map. When a node on an individual map matched one on the emerging combined map, or was added to the combined map for the first time if not already present, the expert's identification number was placed after the node name, as shown by Figure 2. For example, 12 experts mentioned "most recent episode", the top right subconcept in Figure 2. This process allowed each node on the combined map to be traced back to the mind maps of all experts who mentioned it, and from there to the relevant interview transcript lines, thus providing a full audit trail. 
Insert Figure 3 about here

The quantitative analysis of mind maps was achieved by converting the Figure 2 map into one where each node was followed by the total number of different experts who talked about that particular concept (node) or any of its subcomponents, as shown by Figure 3. It indicates that 25 of the 46 experts mentioned some part of "pattern of episodes" as being relevant to risk of suicide, of which 12 were concerned about the most recent episode. Note that the number of experts associated with a node cannot necessarily be determined from the numbers against its subcomponents, because an expert may mention more than one subcomponent, or sometimes only the root concept itself.

The knowledge structures of the combined mind map evolved and were periodically rationalised as subsequent individual mind maps were integrated. The final coding scheme for the combined map imposed a consistent structure on concepts occurring in more than one place. For example, whenever any subcomponent of "pattern of episodes" (Figure 3) is mentioned, such as "most recent episode", the same hierarchical relationship for the subcomponent is recorded; it is placed under "when have episodes taken place" and not directly attached to "pattern of episodes", even if the expert has not explicitly coded the intervening concept. This facilitates comparisons of concepts in different contexts and particularly how experts' perceptions of concepts vary across risks: a major analytic focus in this paper, achieved using the numbers of experts mentioning each mind map node (Figure 3). The validity of the method was checked by two researchers, who recoded some of the transcripts and correctly identified $84 \%$ of the 125 codes associated with those transcripts in the combined mind map.

\section{Validating the content analysis}

Good practice for qualitative data analysis includes participants reviewing analysis of their own interviews, and also the integrated results (Mays \& Pope, 2000). This was achieved 
by distributing the results via the project web site and also by post. No experts queried the accuracy of the mind map representing their own interview's analysis and only minor comments were made about the integrated knowledge structure (i.e. the combined mind map), none raising concerns about its overall legitimacy.

\section{Results}

The comprehensive nature of the combined mind map is indicated by its vital statistics. There are 2,789 nodes across the four risk categories, each representing a particular factor relating to assessment. Only 1,295 of the factors are unique because 534 of them (e.g. depression) occur in more than one place (depression actually occurs in 12 different places). Of the 1,295 uniquely-named nodes, 455 are concept nodes and 840 are leaf nodes. Leaf nodes are the lowest level of data, akin to the measurable cues that underpin risk assessments. The significance of the knowledge hierarchy is that it identifies these cues and their conceptual relationships. Individual risks (e.g. suicide at the left of Figure 3) are linked via a hierarchy of intervening concepts of decreasing abstraction (e.g. "past client episodes of suicide") to the data or cues that provide evidence for them (e.g. "most recent episode").

There is not space to discuss the complete hierarchical structure but Figure 3 illustrates how the method has exposed the detailed knowledge used to assess the pattern of episodes, for example, and the emphases given to its various subcomponents by the collective experts. This paper will concentrate on the most important concepts identified by experts.

Immediate subcomponents of risk

Insert Table 1 about here

Table 1 shows the main risk concepts used by experts (i.e. the first nodes coming off each risk, as partially illustrated in Figure 3 by those concepts directly attached to the suicide node on the far left). Each numbered row quantifies the experts who discussed that particular 
concept. So, for example, life history (Row 3) is relevant to all risks, though only one expert said so for S-N, and the total number of experts who discussed it for any risk is 34 .

Table 1 loosely orders the components of risk by general areas of concern, starting with history, followed by current behaviours, then moving on to concepts relating to mental state and personality, and ending with social, health, and personal details. They will be considered in turn to begin analysing the similarities and differences between factors professionals use to assess the four risks, starting with past episodes of risk (Row 1).

\section{Past episodes of risk}

Insert Table 2 about here

Table 2 displays the main subconcepts involved in assessing past patient episodes (equating to the nodes coming off "past client episodes of suicide" in Figure 3). The first concept is the method used in previous episodes and the table shows planning to be less important for HTO (Row 1.3), suggesting HTO occurs with less forethought and is more dependent on immediate circumstances. It helps explain why "motivation for previous episodes" (Row 5) is associated with suicide and S-H but not HTO and why the pattern of episodes (Row 2) is not so important for HTO, because the lack of premeditation renders it less predictive. On the other hand, the patient's current perspective on previous HTO episodes (Row 4) is significant, because it gives a clue to how resistant patients will be to similar situations in the future.

The external triggers of previous episodes (Table 2, Row 3) relates to triggers in the environment of the patient rather than the patient's behaviour or state of mind. Relationship triggers feature most for suicide and S-H (Row 3.1), which originate externally but are likely to cause changes to the internal emotional and mental state that provide impetus for future actions. However, this connection between internal and external triggers is clearly complex and requires elucidation. Our methods have begun the process but would need to be repeated in more depth. At present, they cautiously suggest a difference between suicide and HTO, with a 
tendency for HTO to be based on reactions to the immediate situation or context within which an episode occurred (Row 3.2); suicide may be motivated more by ongoing circumstances governing emotional and mental states.

\section{Relationship between past episodes of suicide and self-harm}

A number of experts (19) linked past episodes of S-H to risk of suicide in our analysis. It confirmed the difficulties people have with distinguishing suicide and S-H risks, as the literature (e.g. Muehlenkamp, 2005) and a pilot study (Buckingham \& Chan, 2002) indicated. In anticipation of this, a specific question was asked about their similarities and differences. Out of the 36 experts who provided information, 28 said the models of risk were similar and 16 that they were different, which means many said they were both similar and different; a direct indication of the fuzzy boundary. Of the ones saying the risks were similar, 11 said they overlapped, 7 that they were on a continuum, and 4 that $\mathrm{S}-\mathrm{H}$ can lead to suicide. This suggests that assessments of the two risks do overlap, especially where they share subcomponents, but remain distinct, with motivation (11 experts) being the most frequently cited distinguishing factor.

Other history concepts

Returning to Table 1, family history of risk episodes (Row 2) relates mainly to suicide and S-H, but all except S-N are influenced by the patients' general life history (Row 3). A key concern for all three risks is abuse to the patient (Row 3.1), including sexual, physical, and emotional forms, much of which is domestic. Family history of HTO thus seems more relevant if it is turned inwards onto the patients themselves, rather than externally perpetrated.

\section{Current intention to effect risk}

Insert Table 3 about here

Having examined historical issues, the concepts in Table 1 turn to current influences, the 
first being the patient's intention to execute a risk behaviour (Row 4). It applies to all risks except S-N and includes plans and seriousness of intention (Table 3); one might have a well-laid plan but no intention of effecting it. Suicide differs from S-H and HTO because planning and preparation are more important (Table 3, Rows 2.3 and 3.2 in particular). S-H is singled out by an emphasis on current motivation (Row 3.1), the major ones being relief and a cry for help.

\section{Ideation, hallucinations, and delusions}

Intention relates to thoughts clearly connected in the patient's mind to potential actions, whereas ideation (Table 1, Row 5) concerns fantasies about the risk behaviours. Both kinds of thinking were most associated with suicide but also noted in connection with S-H and HTO. Hallucinations and delusions (Table 1, Row 6) refer to confusion about reality and are more associated with HTO, particularly auditory hallucinations (Row 6.1) and paranoid delusions (Row 6.2).

Appearance and behaviour during assessment

Insert Table 4 about here

A person's history, thoughts, and stated intentions are risk indicators that depend on what the person tells the assessor. Assessors also rely on unintentionally relayed signs, which are labelled as "appearance and behaviour during assessment" in Table 1 (Row 7). Most are physical and verbal behaviours, but an interesting addition was the person's effect on the assessor (Table 4, Row 1). Its greater association with suicide and HTO is reflected by the array of feelings induced in the assessor, such as tension, unpredictability, being threatened, hopelessness, anger, horror, and, as one expert put it, "the creeps".

Physical indicators of risk are divided into appearance and body language. Patients' appearance is most useful for assessing risk of S-N (Table 4, Row 2). It was marked by poor personal hygiene (Row 2.1) and general unkempt appearance (Row 2.2), but not body language 
(Row 3), which was considered an indicator of suicide and HTO.

Verbal indicators predicted all risks except S-N (Row 4), but how patients engage with assessors (Row 4.1) is more important than what they actually say. Withholding information is an issue for suicide risk (Row 4.2), although general responsiveness, covering how cooperative and communicative the person is, is associated with all but S-N (Row 4.3). For S-N, what patients look like right now seems more significant for assessors than what they say or do. This is partly predicated by the ability to look after themselves, which is why living skills (Table 1, Row 8) are the domain of S-N, especially related to personal hygiene.

\section{General behaviour and constraints}

What patients are doing outside the assessment, in their current general behaviour (Table 1, Row 9), is also relevant to all risks. Diet was almost exclusively associated with S-N (Row 9.1) and inappropriate sexual, criminal, or abusive behaviour is, as expected, a feature of HTO (Row 9.2). Substance misuse affects all risks (Table 1, Row 10).

Constraints militating against acting out risk behaviours, are most important for suicide (Table 1, Row 11). The primary one is the patient's sense of responsibility and particularly responsibility for the impact of their actions on significant others, which, surprisingly, is only specifically cited by one expert for HTO (Table 1, Row 11.1).

\section{Feelings/emotions and depression}

So far, the concepts discussed have involved patients' history, experiences, and behaviours. The next set examines their general state of mind and personal faculties, starting with feelings/emotions, which Table 1, Row 12 shows was associated with all risks, but especially suicide. An array of different emotions were cited, such as anxiety, distress, feeling neglected, frustration, helplessness, mood swings, negative feelings, and sadness, but the two main ones were anger for HTO and hopelessness for suicide and S-H. Hopelessness was also the emotion most linked to depression (Table 1, Row 13), by 20 experts, and explains the number of experts associating depression with suicide. 


\section{Mental illness}

Mental illness was discussed in all but one interview (Table 1, Row 14), which means the particular sets of cues deemed worthy of diagnostic labels are forming patterns that appear useful for assessing risk. Two concepts of general relevance to mental illnesses were insight into the illness and stage of illness (Rows 14.1 and 14.2). The concern is how patients may cope with knowledge of their mental problems, which relates mostly to suicide and emphasises its reflective aspects. Stage of illness is important because insight is likely to be higher in the early and recovery stages.

The most cited specific diagnoses with respect to risks are bi-polar, psychosis, and schizophrenia, all of which tend to be more associated with suicide and HTO. Schizophrenia emphasises suicide due to the prominence of insight into the illness (Row 14.5.1), continuing the consensus emerging here that suicide has a strong element of internally-oriented reflectiveness. The hypomanic phase of bipolar disorder distinguishes HTO (Row 14.3.1).

Although the underlying cues of mental illnesses are duplicated elsewhere in the risk-assessment knowledge, their combination within particular diagnoses seems to exert a special influence on risk. For example, the cue combinations that lead assessors to think of hypomania predispose them to consider risk of HTO whereas the combinations for schizophrenia are more indicative of suicide. The knowledge hierarchy exposes these patterns, which can be missed by considering cues in isolation.

Attitude, cognitive faculties, personality, and personality disorder

Attitude (Table 1, Row 15) includes patients' insight into their lives and their religious/spiritual beliefs, both almost exclusively associated with suicide (by 10 and 12 experts respectively). Once again, the cognitive and reflective nature of suicide risk is in evidence, further corroborated by the cognitive faculties concept (Table 1, Row 18). On the other hand, impulsiveness (Row 16.1), is the most significant personality factor for all but S-N, probably due to its influence on whether behaviour is acted out, which is relevant to all risks where volition is deemed important. 
Personality disorder (Table 1, Row 17) is one of the few concepts associated with S-H more than other risks. The principal concern is borderline personality disorder (Row 17.1).

\section{Social context and relationships}

Social context (Table 1, Row 19) had twice as many associations with suicide than any other risk. Its two main subconcepts are living arrangements and recent or potential events/changes. Who you live with is more important for suicide than HTO (Row 19.1), underlining the surprising insignificance of potential victims when assessing HTO. Only 12 different experts mentioned victims anywhere in the HTO risk hierarchy, which suggests that the potential for expressing HTO behaviour is more the focus than who might be on the receiving end, and certainly with respect to those sharing living space. For suicide risk, the crucial factor is not who patients live with, but whether they live alone.

Changes in social context are important for both suicide and S-H risks, such as in health and employment, but the most significant change concerns relationships (Row 20.1). Suicide is the most likely response and usually because of the relationship break up or bereavement. This association is repeated for relationships in general (Row 20), where quality of relationships and the extent of external networks are important.

\section{Health issues}

As expected, physical health problems (Table 1, Row 21) are mostly associated with suicide, the emphasis being on pain (Row 21.1) and chronic problems (Row 21.2). With respect to medication or therapies (Row 22), the response to medication is only associated with suicide (Row 22.1), but compliance is important for HTO as well (Row 22.2), presumably because interventions have a constraining effect on risky behaviours.

\section{Demographics}

A person's demographics are mentioned by experts in relation to all risks (Table 1, Row 23), with age and gender being the most cited, followed by ethnicity, with marital status and social class coming some way behind. However, demographic factors are usually cited in 
combination, such as young, single, mothers, and young, single, men; it is powerful justification for considering patterns of cues, not just their individual effects.

\section{Discussion}

Insert Table 5 about here

A coding scheme manifested as a mind map was developed to represent each mental-health expert's risk assessment knowledge elicited during an interview. There was remarkable consistency between the mind maps, justifying further exploration of common structures by combining individual expert's maps into a single integrated one. This facilitated analysis of experts' contributions to each segment of the overall knowledge structure, exposing contrasting emphases between the conceptualisation of individual risks, summarised by Table 5. They can be encapsulated as reflection and forethought for suicide, motivation for S-H, situation and context for HTO, and current presentation for S-N. These will now be considered in more detail, bearing in mind that the results are about how experts organise their risk knowledge; they should not be interpreted as making empirical claims about probabilistic relationships between cues and risk outcomes.

Risk of S-N was distinguished most by a lack of dependence on previous history (patients' or their families'), despite evidence of past neglect being frequently found when it is systematically sought in practice (Dick, Durham, Stewart, Kane, \& Duffy, 2003). Experts also gave minimal consideration to motives or intentions to effect S-N behaviours. Instead, S-N assessment is locked into current circumstances and observable appearance rather than inferences about what is going on mentally or emotionally. These differences may reflect a lower priority placed on assessment of this risk, as well as it being more gradual in onset and expression than the other risks considered. In the expert's view, S-N appears to depend on continuation of adverse circumstances for its detrimental effects, evident from the weight experts placed on patients' appearance, diet, hygiene, care with physical presentation, and living skills. The lack of priority placed on verbal content, body language, feelings, emotions, 
or any assessment of the patients' mental state (apart from depression) indicates assessors do not see S-N as a willed behaviour, comparable to other kinds of S-H. The diminished significance of S-N compared to other risks suggests it is an area in which cognitive modelling needs to be more prescriptive from the outset.

The emphasis placed on patients' intentions in the assessment of the other three risk types implies a more active responsibility on the patients' part that needs to be understood and anticipated over and above the situation in which they find themselves. Insight into patients internal processes is deemed important, as is an examination of past episodes of suicide, $\mathrm{S}-\mathrm{H}$, and HTO, though they are perceived differently in each case. Less emphasis on the degree of planning, the patients' motivation, method used, and the pattern of episodes for HTO means it is seen as located in the immediate context, which may contribute to the notorious difficulty of predicting it from previous episodes. How patients perceive previous HTO episodes is much more important, because it gives a clue about their resistance to behaving in a similar way under repeat circumstances. Such resistance is apparently less significant in assessment for suicide and self harm: suicide is more considered, reflected by the emphasis placed on, for instance, careful planning; $\mathrm{S}-\mathrm{H}$ is linked by assessors to specific but transient motivations such as relief or a cry for help.

When assessing patients' internal emotions and state of mind, suicide and S-H were distinguished from HTO by association with feelings of hopelessness compared with anger. Hopelessness was also a key affective component within depression, thus underpinning the emphasis placed on depression in suicide risk assessment. It is interesting how this contrast between dominance of hopelessness and anger in assessing respectively suicide and HTO mirrors the perceived cognitive difference between suicide and HTO risk: assessment of suicide is influenced by patients' insight into their past, present, and future circumstances (including illnesses) whereas risk of HTO is associated with unthinking impulsivity.

Impulsivity may be why assessors do not pay much attention to how potential perpetrators of HTO view the effects from a victim's perspective. Responsibility for the consequences of behaviour seems more linked to suicide risk and the greater weight placed on 
spiritual or religious beliefs for suicide further reinforces how assessors see suicide as more reflective.

Circumstances surrounding the lives of patients are used in assessing all risks but some elements particularly distinguish one from another. Living alone is most important for suicide risk, but, surprisingly, who one lives with is not an issue for HTO, even though cohabitants are potential victims. Recent events or changes in patients' lives were also only linked with suicide and $\mathrm{S}-\mathrm{H}$, with relationship changes almost wholly related to suicide. It shows how long term situations may impact on the state of mind and thinking of patients for suicide but do not help much with assessing whether their effects will be exhibited by sudden acts of violence.

Overall, the interviews and their analyses reflect the emphases in recent clinical literature of past behaviour (Carter, Reith, Whyte, \& McPherson, 2005; Owens et al., 2005), psychiatric status (Harris \& Barraclough, 1998), and current ideation (Morgan \& Stanton, 1997). One striking addition was clinicians' recognition of their own reactions and affects as an active and valued factor in their assessments, especially for risks of suicide and HTO. While omitted from formal predictive studies, these have been noted by previous observers (e.g. Stengel, 1964; Morgan \& Priest, 1984; Doctor, 2004) and their contribution to risk judgements can be evaluated by the approach described in this paper.

\section{Conclusions}

Our research has generated a comprehensive conceptual hierarchy used by experts in risk assessments and identified data directly corresponding to tangible patient cues. These can be measured with less ambiguity than the judgements required for higher-level concepts, and without the same requirements for mental-health expertise during collection. The cues form the basis of determining what is the relevant information to collect for risk screening tools and in what form. Relevance and importance of risk assessment data is additionally revealed by the method used for eliciting and analysing expertise, which quantified the numbers of experts mentioning each part of the shared knowledge hierarchy. Even if the experts own judgements are occasionally deficient (and our research indicates this may be the case for self neglect), the 
process of externalising and formalising their behaviour highlights areas of improvement. The knowledge structures can be upgraded accordingly and subjected to formal clinical validation within the risk-screening tools, encapsulating best practice in a form that is easy to disseminate through education and training channels.

\section{A decision support system for mental-health risk screening}

The risk-assessment knowledge constructed by our methods will be further refined to become part of a psychological model of classification that represents both the knowledge and reasoning processes of mental-health practitioners (Buckingham, 2002). It will be incorporated within a computerised decision support system able to quantify risk levels associated with individual patients and show how different cues and concepts are influencing assessment. The systematic collection of patient information over time will generate a data resource with the potential for testing the predictive validity of cues. Over time, it will provide invaluable empirical evidence for the decision support system's advice that supplements its judgements based on clinical expertise.

The anchoring of risk information within a psychological model enhances the likelihood of the terms and their relationships being intuitive. This facilitates communication across clinical disciplines and with people lacking a specialist mental-health background, such as those in front-line social and criminal justice agencies. It is of crucial importance for sharing information with patients and carers, who may not have an appropriate risk language of their own (Ryan, 2002).

The eventual aim is to disseminate expertise to front-line services where people may present with mental-health problems but where there may not be anybody with the necessary training to judge the associated risks. The decision support system will provide this expertise so that people's needs can be recognised at an early stage and they can be directed towards appropriate services rather than disappearing off the radar. 


\section{References}

Bouch, J., \& Marshall, J. J. (2005). Suicide risk: structured professional judgement. Advances in Psychiatric Treatment, 11, 84-91.

Buckingham, C. D. (2002). Psychological cue use and implications for a clinical decision support system. Medical Informatics and the Internet in Medicine, 27(4), 237-251.

Buckingham, C. D., \& Chan, T. (2002). Developing a mental-health risk-screening tool (Tech. Rep.). Surrey Hampshire Borders NHS Trust.

Buzan, T. (2003). The mind map boook. BBC Consumer Publishing: London.

Carter, G., Reith, D. M., Whyte, I. M., \& McPherson, M. (2005). Repeated self-poisoning: increasing severity of self-harm as a predictor of subsequent suicide. British Journal of Psychiatry, 186, 253-257.

Castle, K., Duberstein, P. R., Meldrum, S., Conner, K. R., \& Conwell, Y. (2004). Risk factors for suicide in blacks and whites: An analysis of data from the 1993 national mortality followback survey. American Journal of Psychiatry, 161, 452-458.

Dick, P., Durham, T., Stewart, M., Kane, S., \& Duffy, J. (2003). Care programme approach documentation of past risk-related behaviour. Psychiatric Bulletin, 27, 298-300.

Doctor, R. (2004). Psychodynamic lessons in risk assessment and management. Advances in Psychiatric Treatment, 10, 267-276.

Freemind. (2006). www.sourceforge.net/freemind. (url accessed in June, 2006)

Godin, P. M. (2004). 'you don't tick boxes on a form': A study of how community mental health nurses assess and manage risk. Health, Risk \& Society, 6(4), 347-360.

Hanson, R. K. (2005). Twenty years of progress in violence risk assessment. Journal of Interpersonal Violence, 20(2), 212-217.

Harris, E. C., \& Barraclough, B. (1998). Excess mortality of mental disorder. British Journal of Psychiatry, 173, 11-53.

Higgins, N., Watts, D., Bindman, J., Slade, M., \& Thornicroft, G. (2005). Assessing violence risk in general psychiatry. Psychiatric Bulletin, 29, 131-133.

Holdsworth, N., \& Dodgson, G. (2003). Could a new Mental Health Act distort clinical 
judgement? a Bayesian justification of naturalistic reasoning about risk. Journal of Mental Health, 12(5), 451-462.

Maden, A. (2003). Standardised risk assessment: why all the fuss? Psychiatric Bulletin, 25, $129-131$.

Mays, N., \& Pope, C. (2000). Qualitative research in health care: Assessing quality in qualitative research. British Medical Journal, 320, 50-52.

Monahan, J., Steadman, H. J., Appelbaum, P. A., Robbins, P. C., Mulvey, E. P., Silver, E. R., et al. (2000). Developing a clinically useful actuarial tool for assessing violence risk. The British Journal of Psychiatry, 176, 312-319.

Morgan, H. G., \& Priest, P. (1984). Assessment of suicide risk in psychiatric in-patients. British Journal of Psychiatry, 145, 467-469.

Morgan, H. G., \& Stanton, R. (1997). Suicide among psychiatric in-patients in a changing clinical scene. Suicidal ideation as a paramount index of short-term risk. British Journal of Psychiatry, 171, 561-563.

Muehlenkamp, J. J. (2005). Self-injurious behavior as a separate clinical syndrome. American Journal of Orthopsychiatry, 75, 324-333.

Neuendorf, K. (2002). The content analysis guidebook. London: Sage.

Owens, D., Wood, C., Greenwood, D. C., Hughes, T., \& Dennis, M. (2005). Mortality and suicide after non-fatal self-poisoning: 16-year outcome study. British Journal of Psychiatry, 187, 470-475.

Ryan, T. (2002). Exploring the risk management strategies of informal carers of mental health service users. Journal of Mental Health, 11(1), 17-25.

Skegg, K. (2005). Self-harm. Lancet, 366, 1471-1483.

Stengel, E. (1964). Suicide and attempted suicide. Harmondsworth: Penguin.

Szmukler, G. (2001). Violence risk prediction in practice. British Journal of Psychiatry, 178, 84-85.

Watts, D., Bindman, J., Slade, M., Holloway, F., Rosen, A., \& Thornicroft, G. (2004). Clinical assessment of risk decision support (CARDS): The development and evaluation of a 
Risk data 21

feasible violence risk assessment for routine psychiatric practice. Journal of Mental Health, 13(6), 569-581. 


\begin{abstract}
Author Note
The authors wish to thank Gerry Kearns for recruiting and interviewing the experts, as well as helping with the initial data analysis. We are also very grateful to all the mental-health professionals who gave their time to this project. The research was funded by a Department of Health New and Emerging Applications of Technology grant and Ann Adams is supported by a Department of Health NCCRCD Primary Care Career Scientist Award.
\end{abstract}


Table 1

Numbers of experts citing top-level risk components

\begin{tabular}{|c|c|c|c|c|c|c|}
\hline \multirow{2}{*}{\multicolumn{2}{|c|}{ Immediate subcomponents of risks }} & \multicolumn{5}{|c|}{ Number of experts } \\
\hline & & \multirow{2}{*}{$\begin{array}{l}\text { SUI } \\
46\end{array}$} & \multirow{2}{*}{$\begin{array}{l}\mathrm{S}-\mathrm{H} \\
44\end{array}$} & \multirow{2}{*}{$\begin{array}{l}\text { HTO } \\
46\end{array}$} & \multirow{2}{*}{$\begin{array}{l}\text { S-N } \\
31\end{array}$} & \multirow{2}{*}{$\begin{array}{l}\text { SUM } \\
46\end{array}$} \\
\hline & Total experts & & & & & \\
\hline 1 & past episodes of risk & 41 & 36 & 37 & 0 & 46 \\
\hline 2 & family history of risk & 22 & 9 & 4 & 0 & 23 \\
\hline 3 & life history & 25 & 21 & 18 & 1 & 34 \\
\hline & 3.1 abuse to the patient & 14 & 14 & 8 & 0 & 24 \\
\hline 4 & current intention to effect risk & 42 & 28 & 25 & 0 & 46 \\
\hline 5 & ideation about risk & 28 & 13 & 15 & 0 & 33 \\
\hline \multirow[t]{3}{*}{6} & hallucinations and delusions & 10 & 2 & 20 & 4 & 26 \\
\hline & 6.1 auditory hallucinations & 5 & 2 & 11 & 1 & 16 \\
\hline & 6.2 paranoid delusions & 4 & 0 & 13 & 3 & 15 \\
\hline 7 & appearance and behaviour during assessment & 36 & 19 & 25 & 26 & 42 \\
\hline 8 & living skills & 1 & 0 & 0 & 10 & 11 \\
\hline \multirow[t]{3}{*}{9} & current general behaviour & 16 & 9 & 17 & 19 & 32 \\
\hline & 9.1 diet & 1 & 1 & 0 & 18 & 19 \\
\hline & 9.2 inappropriate sexual, criminal, or abusive behaviour & 1 & 1 & 8 & 0 & 8 \\
\hline 10 & substance misuse & 34 & 22 & 35 & 9 & 43 \\
\hline \multirow[t]{2}{*}{11} & constraints on effecting risk & 18 & 4 & 8 & 0 & 25 \\
\hline & 11.1 responsibility for impact on others & 15 & 2 & 1 & 0 & 17 \\
\hline & feelings/emotions & 36 & 22 & 19 & 2 & 42 \\
\hline & 12.1 angry emotions & 2 & 1 & 15 & 0 & 16 \\
\hline & 12.2 hopelessness & 27 & 11 & 1 & 0 & 29 \\
\hline 13 & depression & 45 & 19 & 13 & 17 & 46 \\
\hline
\end{tabular}

Table continues on next page 
Risk data 24

\begin{tabular}{|c|c|c|c|c|c|c|}
\hline \multirow{2}{*}{\multicolumn{2}{|c|}{ Immediate subcomponents of risks }} & \multicolumn{5}{|c|}{ Number of experts } \\
\hline & & \multirow{2}{*}{$\frac{\text { SUI }}{45}$} & \multirow{2}{*}{$\frac{\mathrm{S}-\mathrm{H}}{25}$} & \multirow{2}{*}{$\begin{array}{l}\text { HTO } \\
31\end{array}$} & \multirow{2}{*}{$\frac{\text { S-N }}{14}$} & \multirow{2}{*}{$\frac{\text { SUM }}{45}$} \\
\hline 14 & mental illness & & & & & \\
\hline & 14.1 insight into the illness & 9 & 2 & 1 & 0 & 9 \\
\hline & 14.2 stage of illness & 12 & 2 & 2 & 0 & 13 \\
\hline & 14.3 bipolar & 15 & 7 & 14 & 2 & 22 \\
\hline & 14.3.1 manic/hypomanic phase & 8 & 4 & 13 & 2 & 16 \\
\hline & 14.4 psychosis & 30 & 7 & 23 & 3 & 35 \\
\hline & 14.5 schizophrenia & 25 & 5 & 10 & 2 & 28 \\
\hline & 14.5.1 insight into schizophrenia & 13 & 1 & 0 & 0 & 13 \\
\hline 15 & attitude & 32 & 18 & 16 & 8 & 39 \\
\hline \multirow[t]{2}{*}{16} & personality & 19 & 13 & 21 & 3 & 29 \\
\hline & 16.1 impulsiveness & 16 & 7 & 10 & 0 & 21 \\
\hline \multirow[t]{2}{*}{17} & personality disorder & 5 & 12 & 5 & 0 & 15 \\
\hline & 17.1 borderline personality disorder & 2 & 7 & 1 & 0 & 7 \\
\hline 18 & cognitive faculties & 10 & 3 & 7 & 3 & 15 \\
\hline \multirow[t]{2}{*}{19} & social context & 42 & 21 & 17 & 21 & 45 \\
\hline & 19.1 who client lives with & 16 & 5 & 2 & 3 & 22 \\
\hline \multirow[t]{2}{*}{20} & relationships & 41 & 17 & 20 & 7 & 46 \\
\hline & 20.1 changes to relationships & 27 & 11 & 1 & 1 & 31 \\
\hline \multirow[t]{3}{*}{21} & physical health problems & 25 & 5 & 0 & 9 & 29 \\
\hline & 21.1 pain & 9 & 1 & 0 & 0 & 9 \\
\hline & 21.2 chronic health problems & 21 & 2 & 0 & 3 & 22 \\
\hline \multirow[t]{3}{*}{22} & medication/therapies & 19 & 8 & 10 & 4 & 31 \\
\hline & 22.1 response & 10 & 0 & 0 & 0 & 10 \\
\hline & 22.2 compliance & 6 & 2 & 7 & 3 & 17 \\
\hline
\end{tabular}

Table continues on next page 
Risk data 25

Number of experts

Immediate subcomponents of risks

SUI S-H HTO S-N SUM

23 demographics

$\begin{array}{lllll}31 & 17 & 20 & 7 & 43\end{array}$

The different risks are suicide (SUI), self harm (S-H), harm to others (HTO), and self neglect $(S-N)$; SUM is the sum of different experts mentioning the associated components in any of the risks. The first row, total experts, gives the overall number of different experts who mentioned each risk; indented components are subcomponents of the first one above that is not indented. 
Table 2

Number of experts citing subcomponents of past patient episodes of risk

\begin{tabular}{|c|c|c|c|c|c|}
\hline & \multirow[b]{2}{*}{ Subconcepts of past patient episodes } & \multicolumn{4}{|c|}{ Number of experts } \\
\hline & & SUI & $\mathrm{S}-\mathrm{H}$ & HTO & SUM \\
\hline & Total experts & 41 & 36 & 37 & 46 \\
\hline \multirow[t]{4}{*}{1} & method used in previous episodes & 31 & 22 & 24 & 41 \\
\hline & 1.1 seriousness & 20 & 12 & 9 & 28 \\
\hline & 1.2 type & 15 & 12 & 21 & 33 \\
\hline & 1.3 planning & 9 & 5 & 1 & 9 \\
\hline 2 & pattern of episodes & 25 & 21 & 12 & 33 \\
\hline \multirow[t]{3}{*}{3} & external triggers of previous episodes & 21 & 15 & 17 & 29 \\
\hline & 3.1 relationship triggers & 10 & 9 & 1 & 16 \\
\hline & 3.2 situation/context & 0 & 0 & 8 & 8 \\
\hline 4 & current perspective on previous episodes & 17 & 8 & 12 & 26 \\
\hline 5 & motivation for previous episodes & 15 & 19 & 1 & 23 \\
\hline 6 & end-of-life preparations & 5 & 0 & 0 & 5 \\
\hline
\end{tabular}

The different risks are suicide (SUI), self harm (S-H), harm to others (HTO); SUM is the sum of different experts mentioning the associated components in any of the risks. The first row, total experts, gives the overall number of different experts who mentioned past patient episodes for each risk; indented components are subcomponents of the first one above that is not indented. 
Table 3

Number of experts citing subcomponents of current intention to express risk behaviours

\begin{tabular}{|c|c|c|c|c|c|}
\hline & \multirow[b]{2}{*}{ Subcomponents of current intention } & \multicolumn{4}{|c|}{ Number of experts } \\
\hline & & SUI & $\mathrm{S}-\mathrm{H}$ & HTO & SUM \\
\hline & Total experts & 42 & 28 & 25 & 46 \\
\hline 1 & ability to control self-harming & 0 & 2 & 0 & 2 \\
\hline \multirow[t]{8}{*}{2} & plans & 36 & 10 & 20 & 42 \\
\hline & 2.1 insight into behaviour consequences & 1 & 0 & 0 & 1 \\
\hline & 2.2 length of planning & 1 & 0 & 0 & 1 \\
\hline & 2.3 detail and clarity of plan & 9 & 3 & 1 & 11 \\
\hline & 2.4 prospective method & 31 & 6 & 16 & 37 \\
\hline & 2.5 realism of plan & 2 & 0 & 1 & 3 \\
\hline & 2.6 HTO targets identified & 0 & 0 & 8 & 8 \\
\hline & 2.7 time frame for plan & 1 & 0 & 0 & 1 \\
\hline \multirow[t]{5}{*}{3} & seriousness of intention & 26 & 18 & 8 & 33 \\
\hline & 3.1 current motivation & 4 & 16 & 8 & 22 \\
\hline & 3.2 end-of-life preparations & 16 & 0 & 0 & 16 \\
\hline & 3.3 informed someone about intention & 10 & 1 & 0 & 10 \\
\hline & 3.4 strength of expression of intention & 2 & 1 & 0 & 2 \\
\hline
\end{tabular}

The different risks are suicide (SUI), self harm (S-H), harm to others (HTO); SUM is the sum across all risks. The first row, total experts, gives the overall number of different experts who mentioned current intention for each risk; indented components are subcomponents of the first one above that is not indented. 
Table 4

Number of experts citing subcomponents of appearance and behaviour during assessment

\begin{tabular}{llllll}
\hline & & \multicolumn{5}{c}{ Number of experts } \\
\cline { 2 - 7 } Subcomponents of appearance and behaviour & SUI & S-H & HTO & S-N & SUM \\
\hline \multirow{2}{*}{$\begin{array}{l}\text { Total experts } \\
\text { effect on assessor }\end{array}$} & 36 & 19 & 25 & 26 & 42 \\
2 appearance indicators of risk & 11 & 2 & 10 & 1 & 17 \\
2.1 personal hygiene & 18 & 9 & 8 & 25 & 34 \\
2.2 unkempt & 1 & 0 & 0 & 15 & 16 \\
3 body language & 0 & 0 & 0 & 16 & 16 \\
4 verbal indicators of risk & 17 & 4 & 18 & 0 & 27 \\
4.1 patient engagement with assessor & 18 & 9 & 9 & 1 & 25 \\
4.2 withholding information & 9 & 3 & 3 & 0 & 12 \\
4.3 responsiveness & 12 & 8 & 6 & 1 & 17 \\
\hline
\end{tabular}

The different risks are suicide (SUI), self harm (S-H), harm to others (HTO), and self neglect (S-N); SUM is the combined totals across all risks. The first row, total experts, gives the overall number of different experts who mentioned appearance and behaviour for each risk; indented components are subcomponents of the first one above that is not indented. 


\section{Table 5}

Summary of main differences between the way experts conceptualise risks

\begin{tabular}{|c|c|c|c|c|}
\hline Risk concepts & SUI & S-H & HTO & $\mathrm{S}-\mathrm{N}$ \\
\hline past episodes & $\mathrm{x}$ & $\mathrm{x}$ & $\mathrm{x}$ & \\
\hline planning & $\mathrm{x}$ & $\mathrm{x}$ & & \\
\hline relationship triggers & $\mathrm{x}$ & $\mathrm{x}$ & & \\
\hline situation/context triggers & & & $\mathrm{x}$ & \\
\hline motivation & $\mathrm{x}$ & $\mathrm{x}$ & & \\
\hline pattern of episodes & $\mathrm{x}$ & $\mathrm{x}$ & & \\
\hline family history & $\mathrm{x}$ & & & \\
\hline current intention & $\mathrm{x}$ & $\mathrm{x}$ & $\mathrm{x}$ & \\
\hline planning & $\mathrm{x}$ & & & \\
\hline motivation & & $\mathrm{x}$ & & \\
\hline delusions; auditory hallucinations & & & $\mathrm{x}$ & \\
\hline patient's effect on assessor & $\mathrm{x}$ & & $\mathrm{x}$ & \\
\hline appearance & $\mathrm{x}$ & & & $\mathrm{x}$ \\
\hline hygiene; unkempt & & & & $\mathrm{x}$ \\
\hline body language & $\mathrm{x}$ & & $\mathrm{x}$ & \\
\hline verbal indicators & $\mathrm{x}$ & $\mathrm{x}$ & $\mathrm{x}$ & \\
\hline withholding information & $\mathrm{x}$ & & & \\
\hline responsiveness & $\mathrm{x}$ & $\mathrm{x}$ & $\mathrm{x}$ & \\
\hline living skills and diet & & & & $\mathrm{x}$ \\
\hline inappropriate behaviour & & & $\mathrm{x}$ & \\
\hline sense of responsibility & $\mathrm{x}$ & & $\mathrm{x}$ & \\
\hline awareness of impact on others & $\mathrm{x}$ & & & \\
\hline angry emotions & & & $\mathrm{x}$ & \\
\hline
\end{tabular}




\begin{tabular}{|c|c|c|c|c|}
\hline Risk concepts & SUI & $\mathrm{S}-\mathrm{H}$ & HTO & $\mathrm{S}-\mathrm{N}$ \\
\hline hopelessness & $\mathrm{x}$ & $\mathrm{x}$ & & \\
\hline depression & $\mathrm{x}$ & & & \\
\hline \multicolumn{5}{|l|}{ mental illness } \\
\hline insight into illness; stage of illness & $\mathrm{x}$ & & & \\
\hline bipolar; psychosis; schizophrenia & $\mathrm{x}$ & & $\mathrm{x}$ & \\
\hline bipolar (hypomanic stage) & & & $\mathrm{x}$ & \\
\hline insight into life; religious/spiritual beliefs & $\mathrm{x}$ & & & \\
\hline personality disorder & & $\mathrm{x}$ & & \\
\hline who patient lives with; patient lives alone & $\mathrm{x}$ & & & \\
\hline recent changes in life and/or relationships & $\mathrm{x}$ & $\mathrm{x}$ & & \\
\hline physical health; response to medication/therapy & $\mathrm{x}$ & & & \\
\hline compliance & & & $\mathrm{x}$ & \\
\hline
\end{tabular}

The different risks are suicide (SUI), self-harm (S-H), harm to others (HTO), and self-neglect $(S-N)$. Each cross means the component has a significant association with the risk that distinguishes it from those without a cross for that component; indented concepts are subcomponents of the first one above that is not indented. 


\section{Figure Captions}

Figure 1. Mind map framework used to code risk knowledge from individual interviews.

Figure 2. Part of the fully-expanded "pattern of episodes" concept within suicide risk of the combined map. Numbers after node names represent the identification numbers of different experts who mentioned the node.

Figure 3. Part of the combined-coding mind map for suicide risk, where the numbers after the node names represent the total number of different experts who mentioned that node or any of its subcomponents (nodes within rounded rectangles are concepts without their internal structure displayed). 


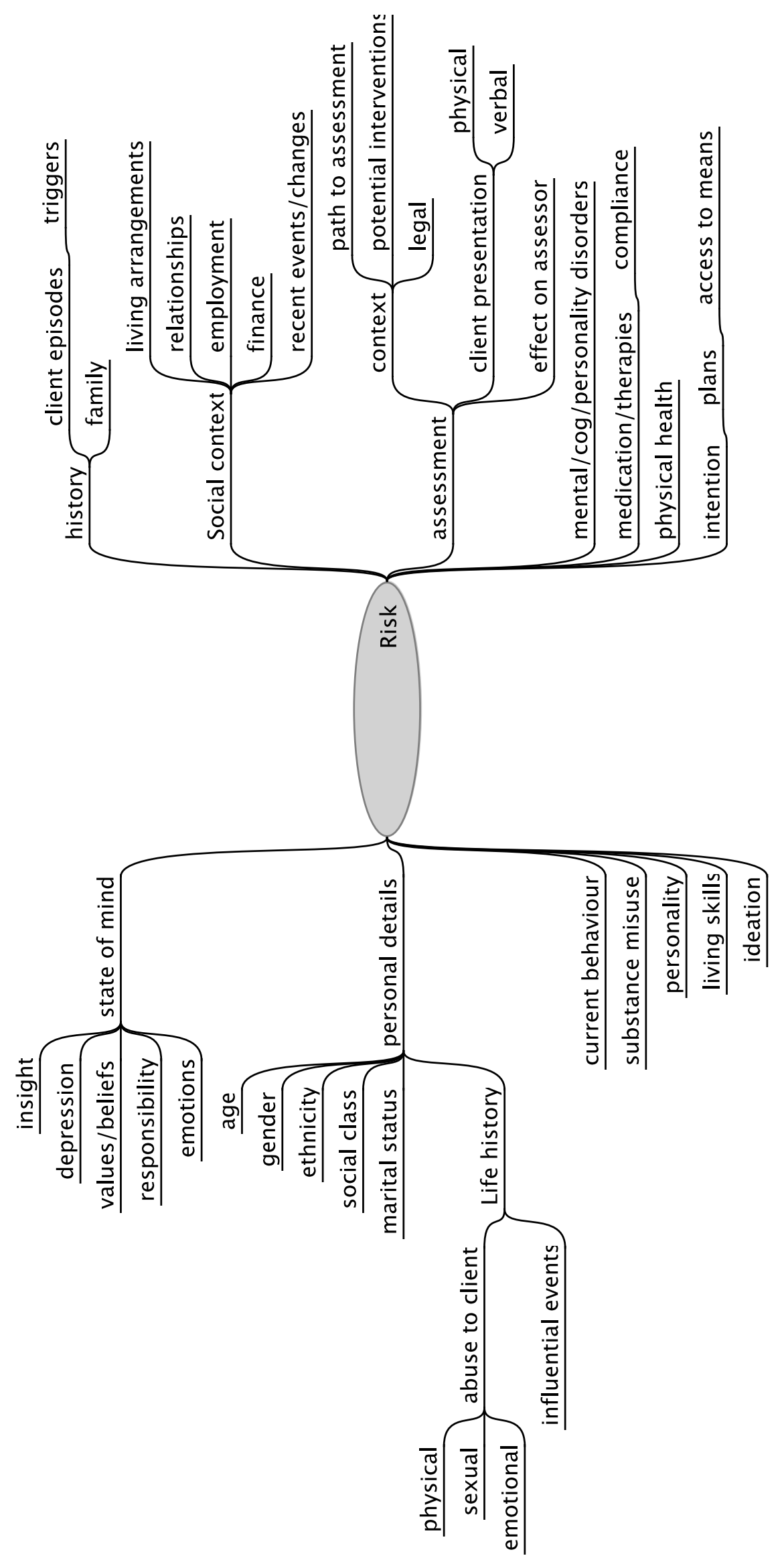




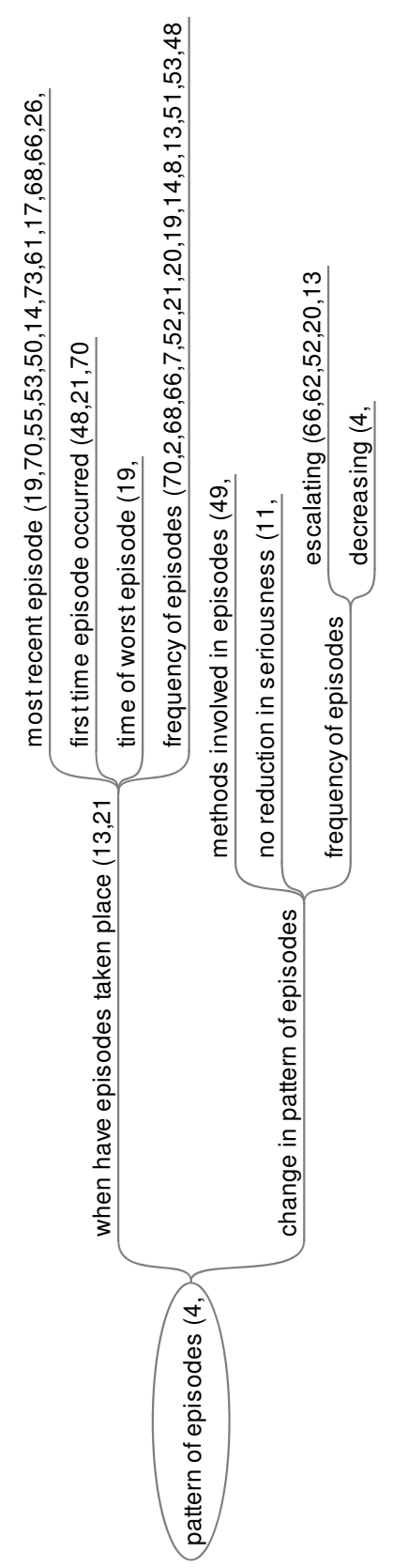




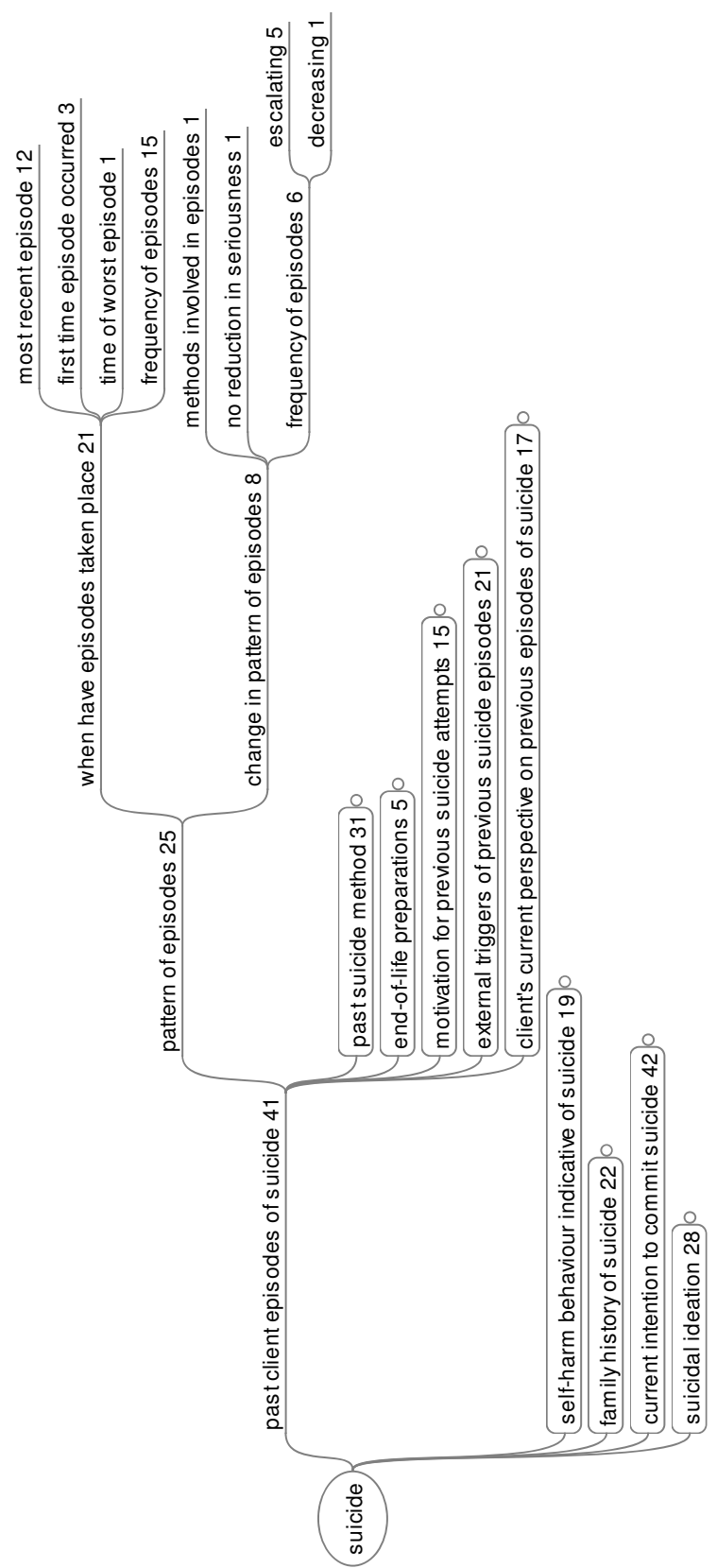

